\title{
Networking im Studium
}

In diesem Aufsatz erfahren Sie, warum Networking eine wesentliche Kompetenz ist und wie Sie ein zuverlässiges Netzwerk aufbauen können. Je früher Sie mit diesen Aktivitäten beginnen, desto besser werden Sie in der Lage sein, interessante Jobs zu finden und Ihre Karriere zu beschleunigen. Die Erfahrung zeigt, dass Menschen, die in ihrem beruflichen Umfeld gut vernetzt sind, erfolgreicher sind und Hindernisse viel leichter überwinden. Um ein guter Netzwerker zu werden, ist es wichtig zuerst zu wissen, was Sie einbringen können, um ein wertvolles Mitglied des Netzwerks zu sein.

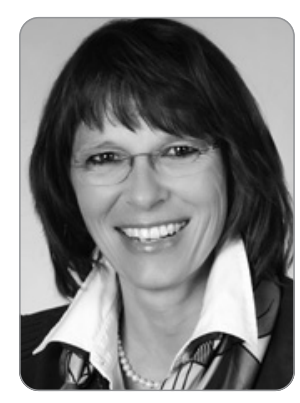

Doris Brenner

ist freie Beraterin mit den Schwerpunkten Personalentwicklung und Karriereberatung (www.karriereabc.de). Sie ist als Gastdozentin an zahlreichen Universitäten und in der Weiterbildung von HR- und Führungskräften in den Fachbereichen tätig.

Summary: This essay will give you an insight on why networking is an essential competence and how you can build up a reliable Network. The sooner you start with these activities the better you will be in the position to find interesting jobs and speed up your career. Experience shows that people who are well connected in their professional environment are more successful and overcome obstacle much easier. To become a good networker it is essential first to know what you can bring in to be a valuable member of the network.

Stichwörter: Networking, Kontakte, beruflicher Erfolg, Jobsuche, Kompetenzen

\section{Was ist Networking?}

Networking, dieser neudeutsche Begriff, vermittelt zunächst den Eindruck, als ob es sich dabei um etwas sehr Innovatives handelt. Quasi ein Kind des 21. Jahrhunderts. Networking ist „in“. Wer nicht netzwerkt, scheint nicht auf dem Stand zu sein. Schaut man sich die zahlreichen Definitionen des Begriffs an, so laufen sie alle darauf hinaus, dass es um den bewussten Aufbau und die Pflege von Kontakten geht. Doch das ist wahrlich nichts Neues. In der ge- samten Menschheitsgeschichte haben sich Individuen bereits zusammengeschlossen und Allianzen gebildet. $0 \mathrm{~b}$ die kirchlichen Orden, die Handwerkerzünfte oder wissenschaftliche Zirkel. Letztendlich gehört es zu den zentralen menschlichen Bedürfnissen, die Gemeinschaft und die Beziehung zu anderen zu suchen.

Was unterscheidet nun das Networking von dem Kontakt in der Familie, dem Freundeskreis oder dem rein geselligen Beisammensein? Beim Networking spielt ein gemeinsames Ziel, ein verbindendes Interesse, eine Zweckbestimmung des Kontaktes eine wesentliche Rolle. Man ist nicht nur zusammen, weil man sich mag oder um nicht alleine zu sein. Das Kontaktnetz wird gezielt gesucht und aufgebaut. Der Zweck oder Nutzen eines Netzwerks besteht darin, dass alle Beteiligten profitieren und durch das Netzwerk gestärkt werden.

\subsection{Zielsetzungen des Networkings}

Für das Networking lassen sich unterschiedliche Zielsetzungen und Wünsche unterscheiden:

- Der Austausch von Informationen. Je mehr Menschen Ihren Wissensschatz einbringen und teilen, umso breiter ist die Datenbasis, je verlässlicher und aussagefähiger die Schlussfolgerung.

- Der Austausch von Erfahrungen. Man muss nicht jeden Fehler selbst machen. So ist es sehr sinnvoll und hilfreich mögliche Fettnäpfchen, in die andere schon getreten sind, zu erkennen und dadurch umgehen zu können.

- Menschen bewusst zusammen bringen. Wo Passgenauigkeit herrscht und gemeinsame Interessen bestehen, lässt sich besser und zielgerichteter arbeiten und sich auch Synergien erzielen.

- Gemeinschaft spüren. Besonders in schwierigen Zeiten und in Veränderungsprozessen, wie der Existenzgrün- 
dung oder der Jobsuche ist es essenziell gut eingebunden zu sein und Rückhalt zu spüren.

- Interessen und Anliegen zusammen vertreten. Gemeinschaft stärkt, schafft mehr Aufmerksamkeit und erhöht die Ressourcen. So lassen sich Positionen und Interessen mit mehr Gewicht nach außen vertreten und auch durchsetzen.

- Ehrliches Feedback und Rat. Reflexion und persönliche Weiterentwicklung ist nur möglich, wenn auch von außen verlässliche Rückmeldungen kommen. Dies setzt voraus, dass Sie diesen Menschen vertrauen und sich auf deren Expertise und Einschätzung verlassen können.

Ein ganz zentraler Faktor des Networkings ist das gerade im letzten Punkte genannte gegenseitige Vertrauen, die Verlässlichkeit. Letztendlich basiert das gesamte System wie bei einer Kette darauf, dass jedes Glied seinen Beitrag zur Stabilität leistet und damit erst der Nutzen für alle Beteiligten entsteht.

\subsection{Kriterien für Netzwerke}

Eine solide Basis stellen Netzwerke mit Menschen dar, mit denen Gemeinsamkeiten, insbesondere Werte und Einstellungen geteilt werden. Am besten ist es, Netzwerke in guten Zeiten aufzubauen und zu pflegen, wenn man sie nicht wirklich braucht. Nur dann werden verlässliche, vertrauenswürdige Partner gerade auch in schwierigen Zeiten zur Seite stehen. Hier zeigt sich, dass gerade Kontakte aus dem Studium eine hohe Tragfähigkeit haben können. Dabei ist nicht in erster Linie die Quantität als vielmehr die Qualität entscheidend. Exklusivität im Zugang und in der begrenzten Zahl der Netzwerkmitglieder kann oft als Qualitätskriterium gesehen werden. So sind Netzwerke von Preisträgern eher elitär aufgebaut. Geht es um die Vertretung von Interessen nach außen kann dagegen eine große Zahl von Mit- gliedern oder Partnern sehr sinnvoll sein und die notwendige Power bringen.

\section{Was sollte ein guter Netzwerker mitbringen?}

Häufig wird als zentrale Anforderung an einen guten Netzwerker eine ausgeprägte Kommunikationsfähigkeit und Extrovertiertheit genannt. Diese Eigenschaften sind sicherlich vorteilhaft. Weitaus wichtiger zeigt sich doch in der Praxis, dass Netzwerker einen Mehrwert für die jeweilige Gruppe darstellen sollten. Daher ist eine realistische Bestandsaufnahme der eigenen Kompetenzen, Fähigkeiten und Erfahrungen in diesem Zusammenhang sehr hilfreich. Diese stellen die Grundlage dar, um als Teammitglied einen Mehrwert bieten und damit die Ziele des Netzwerkes aktiv verfolgen zu können (vgl. Abb. 1).

\section{- Fachkompetenz:}

Hier sind Wissen und Kenntnisse gefragt. Während des Studiums werden diese vorwiegend in dem jeweiligen Studienfach angesiedelt sein. Fachkompetenz kann jedoch auch über Hobbys, Ehrenamt oder Nebenjobs erworben werden.

Fachkompetenz bietet die Möglichkeit, Informationen weitergeben, andere inhaltlich beraten und Zusammenhänge aufzeigen zu können.

\section{- Methodenkompetenz:}

Die Herangehensweise bei der Lösung von Aufgaben steht dabei im Mittelpunkt. Methodenkompetenz stellt eine Werkzeugbox dar, die sich themenunabhängig anwenden lässt. Projektmanagement, Präsentationstechniken, oder Bewertungsverfahren sind hier beispielhaft zu nennen.

Methodenkompetenz bietet die Möglichkeit, Aufgaben im Netzwerk strukturiert und zielgerichtet bearbeiten $\mathrm{zu}$ können.

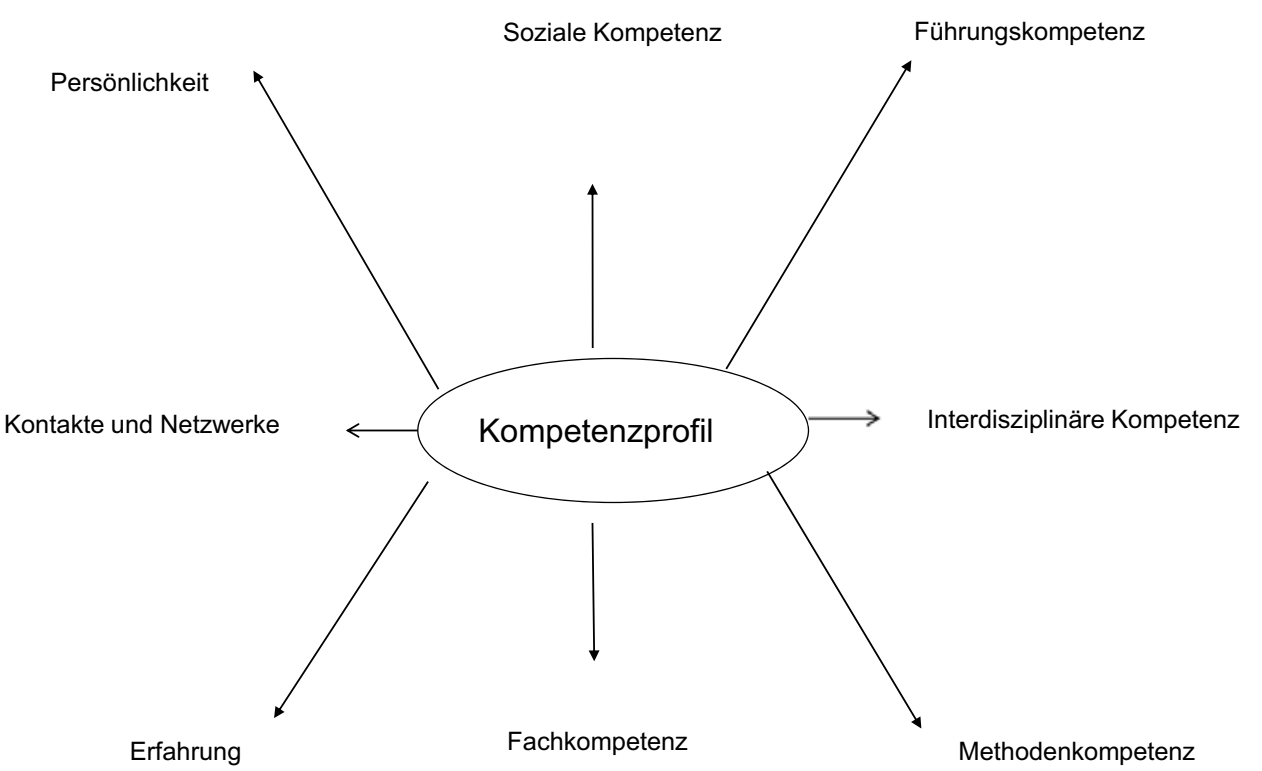

Abb. 1: Kompetenzprofile 


\section{- Soziale Kompetenz:}

Der situationsgerechte Umgang mit oft sehr unterschiedlichen Menschen ist ein zentraler Aspekt dieser Kompetenz. Gerade im Networking ist dies besonders gefragt. Wer die Bedürfnisse anderer Menschen erkennt, vermittelnd bei Meinungsverschiedenheiten agiert und $\mathrm{zu}$ einem positiven Arbeitsklima beiträgt, kann einen wichtigen Beitrag leisten.

\section{- Führungskompetenz:}

Sie ist eng mit der sozialen Kompetenz verbunden erfordert jedoch zusätzlich eine Zielorientierung sowie die Fähigkeit Potenziale bei Menschen zu erkennen und die passenden Teams zusammenzustellen. Leitungsaufgaben in einem Ehrenamt oder bei Freizeitaktivitäten bieten auch Studenten eine Möglichkeit hier erste Erfahrungen zu sammeln.

Wer Führungskompetenz einbringen kann, wird die Erreichung der im Netzwerk formulierten Ziele maßgeblich fördern.

\section{- Interkulturelle Kompetenz:}

Wer sich in unterschiedlichen Kulturen zu Recht finden kann und verschiedene Sprachen spricht, ist gut gerüstet für die Arbeit in unserer immer globaler werdenden Welt. Gerade Studenten bewegen sich häufig in einem internationalen Umfeld, sei es durch Auslandssemester, globale Projekte oder ausländische Kommilitonen.

Interkulturelle Kompetenz erleichtert das Miteinander in internationalen Netzwerken und hilft dabei Menschen zusammenführen und Toleranz für die Andersartigkeit zu schaffen.

\section{- Interdisziplinäre Kompetenz:}

Die Fähigkeit mit Menschen unterschiedlicher Fachgebiete zusammenarbeiten $\mathrm{zu}$ können und offen $\mathrm{zu}$ sein für deren spezifische Prioritäten und Herangehensweisen, ist in der komplexen Arbeitswelt ständig gefordert. Wirtschaftsingenieure beispielsweise zeichnet dies explizit aus.

Netzwerker mit dieser Fähigkeit können Brücken bauen und zwischen verschiedenen Fachdisziplinen vermitteln und übersetzen.

\section{- Erfahrung:}

Mittels Erfahrung lässt sich nachweisen, dass bestimmte Kompetenzen und Fähigkeiten nicht nur theoretisch vorhanden sind sondern diese auch in der Praxis zielgerichtet zur Erreichung von Zielen bereits eingesetzt werden konnten. Da Studenten nur begrenzt über klassische Berufserfahrung verfügen, können sie verstärkt über Praktika, Ehrenamt oder Freizeitaktivitäten Erfahrung vorweisen.

Menschen mit Erfahrung können vor unnötigen Fehlern in der Umsetzung eines Projektes bewahren, Best Practices aufzeigen und für das Gefühl von Sicherheit in einem Netzwerk sorgen. Gerade die Netzwerkarbeit selbst bietet hier ein hervorragendes Übungsfeld.

\section{- Persönlichkeit:}

Die Wesenszüge eines Menschen stellen einen entscheidenden Faktor im Zusammenhang mit der Netzwerkarbeit dar. Hilfsbereitschaft, Toleranz, Begeisterungsfähigkeit oder auch Verantwortungsbereitschaft sind nur einige Beispiele, die in einem Netzwerk gefragt sind.

In diesem Zusammenhang sind auch die Werte zu nennen, die einem Netzwerk als gemeinsame Basis aller Mitglieder zugrunde liegen. Nur wer sich mit diesen identifizieren kann, wird sich auf Dauer in dem Netzwerk zuhause fühlen.

\section{- Kontakte und Netzwerke}

Auf der einen Seite stellen die genannten Kompetenzen eine wichtige Basis dar, um ein guter Netzwerker zu sein. Gleichzeitig stärken Kontakte und Netzwerke das eigene Qualifikationsprofil. Wer über ein gutes fachliches Netzwerk verfügt, kann bei der Aufgabenbewältigung auf einen reichen Fundus an zusätzlicher Expertise zurückgreifen. Gerade Studenten sollten sich darüber bewusst sein, dass sie mit ihren vorhandenen Kontakten zu Professoren und Kommilitonen an der Hochschule, und dem Wissen über dortige Strukturen, für Arbeitgeber über interessante Zugangsmöglichkeiten im Hinblick auf Kooperationen oder auch die Rekrutierung von Nachwuchskräften verfügen.

\section{Welche Netzwerke bieten sich an?}

Netzwerke lassen sich in unterschiedliche Kategorien einteilen.

\subsection{Parteien/Verbände/formelle Institutionen}

Wenn es darum geht gesellschaftliche Themen voran bringen zu wollen, bzw. selbst Einfluss ausüben zu wollen, sollte ein Engagement in erster Linie bei formellen Institutionen wie Parteien und Verbänden erfolgen. Hier geht es darum Interessen zu bündeln, die Position einer Interessengruppe zu stärken und ein Thema nach vorne zu bringen. Je nachdem, wo die spezielle Zielrichtung liegt, gilt es zu überlegen, sich eher regional oder überregional zu organisieren oder einzubringen.

\subsection{Berufliche Netzwerke und Fachveranstaltungen}

Wenn der fachliche Erfahrungsaustausch bzw. die Weiterbildung im Vordergrund stehen und der Kontakt mit Menschen "gleicher Wellenlänge" gesucht wird, bieten sich Berufsverbände, wissenschaftliche Gesellschaften, Arbeitsgruppen oder auch Fachveranstaltungen wie Tagungen oder Messen besonders an. Auch Fachforen im Internet können hier hilfreich sein. Für Studenten bieten Berufsver- 
bände meist sehr günstige Sonderkonditionen was die Mitgliedsbeiträge betrifft.

\subsection{Individuelle Kontakte}

Einzelkontakte, persönliche Weiterentwicklung und vertrauensvoller Austausch lassen sich über informelle Netzwerke und individuelle Kontakte sehr gut realisieren. Sie basieren häufig auf einer 1:1 Beziehung. Mentoring- oder Coachingprogramme können hier hilfreich sein. Oft ergeben sich intensive individuelle Beziehungen auch aus formellen Netzwerken.

\subsection{Eigene Netzwerke gründen}

Sofern es für relevante Themen oder Interessen noch keine Netzwerke gibt, kann es sinnvoll sein, diese selbst ins Leben zu rufen. Dies kann eher informell in Gesprächskreisen oder Chats oder auch mit Strukturen wie in einem Verein erfolgen.

\subsection{Virtuelle Netzwerke}

Heute stehen zahlreiche virtuelle Networking Portale zur Verfügung. Im Gegensatz zu Facebook sind LinkedIn und Xing berufliche Netzwerkplattformen. Sie machen es einfach mit einer großen Zahl von Menschen in Kontakt zu treten und auch deren Veränderungen wie Jobwechsel jederzeit erkennen zu können. Ferner bieten sie eine gute Plattform, um am Arbeitsmarkt wahrgenommen zu werden, was insbesondere bei der Jobsuche wichtig ist. Dies ist sehr positiv zu bewerten. Doch gerade in der Unverbindlichkeit des www ist die Verlässlichkeit und Seriosität der Informationen und Kontakte nicht immer gegeben. Und genau hier treffen virtuelle Netzwerke und Portale oft an ihre Grenzen.
Daher gelten in die folgenden Empfehlungen:

- Pflegen Sie Ihr Profil und achten Sie darauf, wer welche Informationen sehen kann

- Überlegen Sie sich, mit wem Sie sich verlinken. Ihre Verbindung zu einer Person kann als Referenz verstanden werden und Sie bürgen mit Ihrem Ruf.

- Nicht die Quantität der Kontakte sollte im Vordergrund stehen sondern die Qualität.

- Versuchen Sie virtuelle Kontakte möglichst auch durch persönliches Kennenlernen zu festigen

- Eine wertschätzende seriöse Ansprache sollte auch in der virtuellen Welt selbstverständlich sein

\section{Networking bei der Jobsuche}

Gerade bei der Jobsuche spielt Networking eine entscheidende Rolle, da der überwiegende Teil der Stellen über den sogenannten verdeckten Arbeitsmarkt vergeben wird (vgl. Abb. 2).

Aus Sicht eines Arbeitgebers besteht das geringste Risiko bei einem Kandidaten, der bereits im Unternehmen tätig war und damit gemeinsame Arbeitserfahrung vorliegt. Neben festen Mitarbeitern gehören zu dieser Gruppe auch Praktikanten, Werkstudenten oder freie Mitarbeiter. Hier zahlt es sich aus, bereits während der Studienzeit Praxiserfahrung zu sammeln und damit den Fuß in der Tür zu haben.

Wer über ein gutes Netzwerk verfügt, erfährt bereits von Stellen, die noch nicht offiziell ausgeschrieben sind oder wird sogar direkt angesprochen. Viele Unternehmen bitten ihre Mitarbeiter ganz konkret um Kandidatenvorschläge. Daher ist der Kontakte zu ehemaligen Studienkollegen hier eine gute Möglichkeit ins Gespräch gebracht zu werden. Kontakte lassen sich auch sehr gut auf Fach- oder Rekrutierungsmessen knüpfen. Bei der Kontaktaufnahme wird die eigene kurze Vorstellung von entscheidender Bedeu-

\section{Rekrutierungswege}

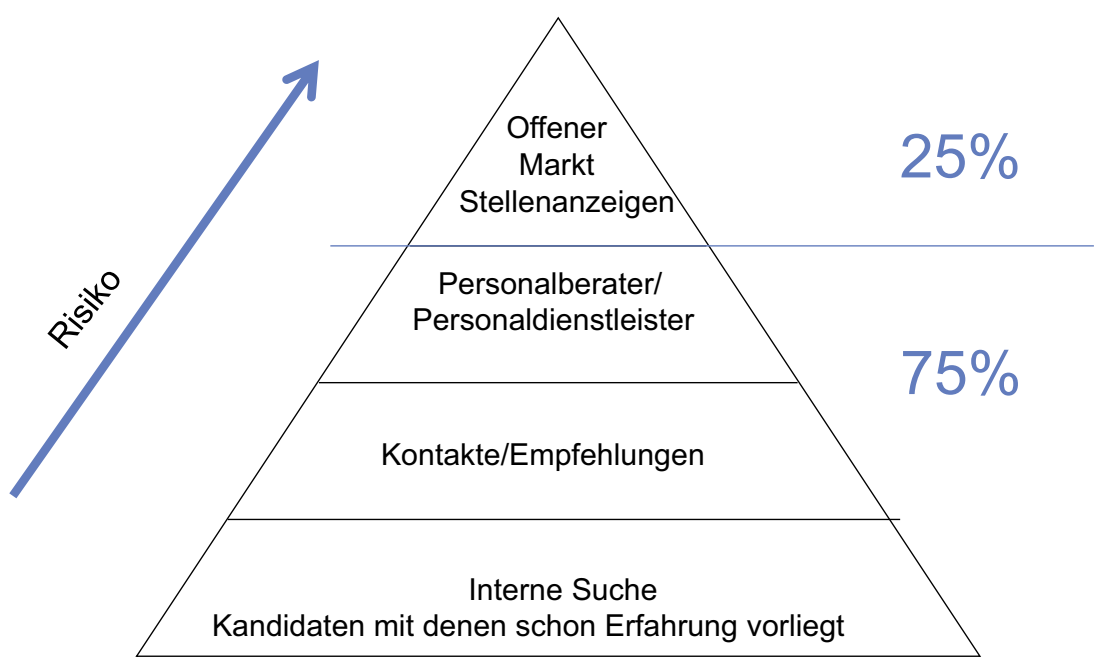

Abb. 2: Rekrutierungswege 
tung sein. Es geht darum, dem Gesprächspartner möglichst prägnant den eigenen Hintergrund zu vermitteln und konkrete, interessante Ansatzpunkte aufzuzeigen. Für eine solche Kurzvorstellung hat sich der englische Begriff Elevator Pitch etabliert. Es steht also als Orientierung die Zeit einer Fahrt mit dem Aufzug zur Verfügung, und bewegt sich zwischen 10 und 50 Sekunden.

Auch über die Mitgliedschaft in Berufsverbänden lassen sich, insbes. wenn während des Studiums nur wenig Praxisbezug bestand, Kontakte sehr gut knüpfen. Einschlägige Veranstaltungen wie Vorträge oder Arbeitskreise bieten hierzu Raum.

\section{Ins Gespräch kommen - Smalltalk}

Oft empfinden Menschen es als schwierig ins Gespräch zu kommen und einen Einstieg zu finden. Wörtlich übersetzt ist der Small Talk einfach eine kleine Unterhaltung. Sie sollte etwas Leichtes und Lockeres an sich haben und Lust auf mehr machen. Und genau das ist der Ansatzpunkt, wenn es darum geht, mit einer fremden Person ins Gespräch zu kommen.

Natürlich könnte man gleich „zur Sache kommen“. Das wirkt oft plump, unhöflich und ist wahrlich kein Einstieg, der Sympathie schaffen wird.

Der Small Talk erfüllt also die Funktion, des zwanglosen sich Annäherns, des sich Herantastens. So paradox es klingt, aber damit ist der Small Talk gerade für schüchterne Menschen sehr hilfreich. Er bietet die Möglichkeit, die eigene Schutzzone und die des anderen zu wahren und sich vorsichtig $\mathrm{zu}$ beschnuppern. Small Talk verpflichtet $\mathrm{zu}$ nichts, er eröffnet jedoch die Möglichkeit für mehr.

Nun stellt sich die Frage des "Wie".

Es gibt eine ganze Reihe von Möglichkeiten, mit einem Menschen ins Gespräch zu kommen. Der einfachste Weg ist über Gemeinsamkeiten. Bei einer Tagung und Konferenz verbindet auf jeden Fall das Veranstaltungsthema.

"Da war ja ein großer Andrang bei diesem Vortrag. Wie ist denn Ihr Eindruck?"

Die offene Frage nach dem persönlichen Eindruck bietet dem Gegenüber alle Freiräume der Antwortmöglichkeit. Möchte er ins Gespräch kommen, wird er einsteigen und erzählen, hat er gerade keine Lust oder ist in Eile, kann er kurz antworten und sich dann wieder entfernen.

Auf Verbandstreffen oder bei Netzwerkveranstaltungen kann auch die Frage: "Ich sehe Sie hier zum ersten Mal, was hat Sie hergeführt?" ein Einstieg sein.

Oft ergeben sich erste Gesprächsmöglichkeiten in den Pausen, bei einem Kaffee oder am Buffet. Damit ergibt sich ein guter Einstieg, um ins Gespräch zu kommen. „Ein guter Kaffee ist jetzt genau das, was ich brauche. Darf ich Ihnen auch eine Tasse einschenken?"
Wer aufmerksam ist, findet wesentlich leichter ins Gespräch. "Möchten Sie auch etwas Milch in Ihren Kaffee" Freundliche Gesten der Hilfe sind Sympathieträger und machen den Gesprächseinstieg leichter.

Etwas Nettes über sein Gegenüber oder das Ambiente zu sagen, schafft ebenfalls einen guten Einstieg. Ernst gemeinte Komplimente sind in der Tat die besten Türöffner im Small Talk. „Was für ein herrlicher Blumenstrauß.“ Doch Vorsicht! Anbiedernde Schmeichelei wirkt meistens aufgesetzt und bewirkt genau das Gegenteil, von einem positiven Gesprächsstart. Beide Gesprächspartner sollten sich wohl fühlen, damit eine gemeinsame Schwingung entstehen kann. Auch gemeinsames Lachen kann dazu beitragen und ist ein guter Einstieg. Eine witzige Bemerkung, eine Geste oder Mimik kann das Eis brechen und beweist Humor. Ergeben sich aus dem Small Talk ganz konkrete Ansatzpunkte für einen weiteren Kontakt und beide Gesprächspartner drücken ein Interesse aus, das Gespräch zu vertiefen, so ist es wichtig, möglichst konkret die weiteren Schritte zu vereinbaren. Small Talk lässt sich wunderbar in allen möglichen Situationen üben. Sei es in der Bahn oder in der Schlange vor der Kinokasse. Je ungezwungener die Situation, umso besser eignet sie sich als Übungsfeld.

Hier noch zum Abschluss ein kleiner Knigge zum Thema Small Talk:

- Achten Sie auf die Leichtigkeit und Zwanglosigkeit beim Einstieg in den Small Talk.

- Eine freundliche Ausstrahlung, ein Lächeln erleichtern den Kontakt

- Themen wie Krankheiten, Religion, Politik und natürlich alles Diskriminierende gehören nicht hier her

- Halten Sie mit Ihrem Gesprächspartner Blickkontakt und schenken Sie ihm Ihre volle Aufmerksamkeit.

- Setzen Sie Ihren Gesprächspartner nicht unter Druck mit einem Anliegen. (Häufiges Problem bei Rechtsanwalt, Steuerberater oder Arzt. "Wenn ich Sie gerade sprechen.....)

- Texten Sie Ihren Gesprächspartner nicht zu, achten Sie auf Ausgewogenheit

- Nutzen Sie offene Fragen (woher, wozu, weshalb, wie...) um den Gesprächsfluss in Gang zu bringen

- Finden Sie Gemeinsamkeiten, das schafft Verbindung und inhaltliche Themen

- Small Talk heißt kleine Unterhaltung. Belassen Sie es dabei und nehmen Sie den Gesprächspartner nicht in Beschlag. Bei konkreten Ansatzpunkten verabreden Sie sich lieber für eine Vertiefung.

\section{Literatur}

Brenner, D., Networking im Job, Freiburg 2017.

Brenner, D., Punkten Sie mit Ihren Stärken, Freiburg 2015.

Brenner, D., Karrierestart nach dem Studium, Freiburg 2015. 